\title{
The role of primary care in management of rare diseases in Ireland
}

\section{Niall Byrne ${ }^{1} \cdot$ Jacqueline Turner $^{1} \cdot$ Rita Marron $^{1} \cdot$ Deborah M. Lambert $^{1}$ (D) $\cdot$ Daniel N. Murphy ${ }^{1} \cdot$ Grace O'Sullivan $^{1,2}$. Maureen Mason ${ }^{1}$. Frank Broderick ${ }^{3} \cdot$ Mary C. Burke $^{4} \cdot$ Sheila Casey $^{5} \cdot$ Marguerite Doyle $^{6} \cdot$ David Gibney $^{7}$. Fergus Mason ${ }^{8} \cdot$ David Molony $^{9} \cdot$ Deirdre Ormond $^{10} \cdot$ Colm O' Sé $^{11} \cdot$ Conor O'Shea $^{12} \cdot$ Eileen P. Treacy $^{1,2}$}

Received: 4 December 2019 / Accepted: 24 December 2019 / Published online: 13 January 2020

(C) The Author(s) 2020

\begin{abstract}
Background 'Slaintecare' aims to address complex patient care needs in an integrated fashion with an emphasis on patientcentred, patient-empowered community care.Currently there is a lack of knowledge of the impact of rare disease management in primary care and of the information tools required by general practitioners to deliver integrated care for rare disease patients. Aims To complete a pilot survey to estimate the general practice clinical workload attributable to selected rare diseases and assess the use of relevant information sources.

Methods A retrospective cross-sectional survey was carried out of general practice consultations (2013-2017) for patients with 22 commonly recognised rare diseases.

Results Around 31 general practitioners from 10 Irish practices completed information on 171 patients with rare diseases over 3707 consultations. General practice-specific coding systems were inadequate for rare disease patient identification. Over 139 $(81.3 \%)$ patients were adult, and $32(18.7 \%)$ were children. Management of care was hospital and not primary care based in $63 \%$. Those eligible for state-reimbursed care had a significantly higher median number of consultations ( 23 consultations, IQR $=13-$ 37 , or 5.8 consultations/year) than those who paid privately (10 consultations, IQR $=4-19$, or 2.5 consultations/year) $(p<0.005)$. General practitioners had access to public information resources on rare diseases but few had knowledge of (35.5\%), or had ever used (12.9\%) Orphanet, the international rare disease information portal.

Conclusions Both specific rare disease-specific coding and use of the relevant rare disease information sources are lacking in general practice in Ireland.
\end{abstract}

Niall Byrne and Jacqueline Turner both authors are joint first authors as they contributed equally to the manuscript.

Eileen P. Treacy

etreacy@mater.ie

1 National Rare Disease Office, Eccles St, Dublin D07 R2WY, Ireland

2 National Clinical Programme for Rare Diseases, HSE and RCPI, Dublin, Ireland

3 Fairview Medical Centre, Fairview Strand, Dublin, Ireland

4 Glasnevin Family Practice, Finglas Road, Glasnevin, Dublin, Ireland

5 Kingscourt Surgery, Kingscourt, Co. Cavan, Ireland

6 Aubrey Medical Centre, Shankill, Dublin, Ireland

7 Ballymun Family Practice, Ballymun, Dublin, Ireland

8 Johnstown Medical Centre, Cabinteely, Dublin, Ireland

9 The Red House Family Practice, Mallow, Co., Cork, Ireland

10 Windmill Medical Centre, Skerries, Co., Dublin, Ireland

11 Ballyfermot Medical Centre, Dublin, Ireland

12 Wheaton Hall Medical Practice, Co Louth, Drogheda, Ireland
Keywords Clinical coding · General practitioners · Integrated delivery of healthcare - Patient care management - Rare diseases

\section{Background}

In Europe, a disease is considered rare if it occurs in less than 5 per 10,000 persons. Rare diseases (RDs) are defined as life-threatening or chronically debilitating diseases with a high level of complexity. Most RDs are associated with chronic physical, intellectual or neurological disabilities. Collectively, they pose a significant economic burden [1-3]. The greater than 6100 known RDs are estimated to affect $3.5-5.9 \%$ of the population (excluding rare cancers and infectious diseases) equating to 263-446 million persons globally [4] and approximately 300,000 Irish people. $72 \%$ of RDs are genetic and $75 \%$ of RDs have paediatric onset [4]. However, the true prevalence of only a few rare disease in Ireland is unknown as there is currently no national RD registry and there are 
insufficient coding systems in place to capture RD prevalence [3]. Patients affected with RDs frequently describe a prolonged 'diagnostic odyssey' and significant difficulties in accessing coordinated care and treatment $[1,5-7]$.

The expansion of genomic testing will accelerate and expand the diagnosis of RDs [8]. With these developments and increased introduction of the 'personalised care' approach, there will be a concomitant need to develop clinical guidelines, clinical pathways, clinical decision support tools and patient information tools that are easily accessible at the point of care and that will empower patients in self-management $[8,9]$.

Adequate care coordination is particularly important for those affected by rare conditions. The UK 2011 National Coalition on Care coordination defined care coordination as 'a person-centred, assessment-based, interdisciplinary approach to integrating health care and social support services in a cost-effective manner, in which an individual's needs and preferences are assessed, a comprehensive care plan is developed and services managed and monitored by an evidencebased process'. Such coordination may be necessary, for example, when care is required over a long period of time; the level of care required increases; multiple services are needed; or a high frequency of emergency unplanned admissions occurs. For RDs, coordination across services is often essential and likely includes health, social, primary, secondary, tertiary and quaternary care, as well as voluntary sectors. Many patients will present with symptoms for the first time to their general practitioner (GP). Furthermore, most families of RD patients agree that their GP is in the position to coordinate the extensive range of services needed $[10,11]$.

Primary care can provide accessible and affordable care to RD patients and thereby reduces hospital admissions. GPs can guide RD patients through complicated hospital systems and coordinate care, potentially reducing both patient hardship and costs of care. Primary care teams offer psychological support to patients and their families and advocate access to expert healthcare in the community setting $[11,12]$. In Ireland, state-reimbursed GP care is available to all people under 6 years or over 70 years or to people between 6 and 70 who qualify for a means-tested medical card; all others must privately pay for GP services.

The long-term plan for the UK NHS is to shift its emphasis from acute care hospitals to a new service model based on community care, population health and collaboration [13]. The Irish 'Slaintecare' plan also emphasises the enhanced integrated care of patients with chronic diseases in the community [14].

Primary care providers/general practitioners have a central role in health promotion and disease prevention. The National Clinical Programme for Rare Diseases has developed a model of care for RDs to improve access, value and quality of care for RD patients (https://www.lenus.ie/handle/10147/626904). This document highlights the unique role of the GP in coordinating care for RD patients. Knowledge of the underlying disorder and care pathway is crucial for the community treatment of individuals with a RD.

Orphanet is the globally used Europe-based website providing comprehensive information about orphan drugs and rare diseases, including information summaries, clinical practice guidelines and information about the location of expert resources, clinical research and trials, used by both physicians and patients. The website is managed by a consortium of academic establishments from 35 countries, led by Inserm (www.orpha.net) [15]. Ireland participates in the Orphanet consortium, and the Orphanet Ireland team based at the National Rare Diseases Office collects and curates information about national RD resources for the Orphanet database.

To date, there are no existing studies that explore the services provided by Irish general practice for people with RDs or the GPs' use of RD resources. In this pilot survey, we explored the role of Primary Care/GPs in the Irish RD patient care journey.

\section{Methods}

\section{Survey design}

The survey was designed by National Rare Diseases Office (NRDO) staff to assess general practice involvement in the management of RD patient care in Ireland over a 4-year period (from June 2013 to June 2017). Documents produced for this survey included a list of 22 rare diseases with corresponding ICD-10 codes; a survey of the use of rare disease resources in primary care practices; and a questionnaire to be completed by the GP regarding each patient identified with one of the 22 rare diseases. The target of the survey was GPs registered with the Irish Medical Council or holding a place on the GP training scheme, in any size general practice, and in any county of Ireland.

About 22 RDs were chosen for survey on the basis of their relative high prevalence, chronic course and recognisability (Table 1). This survey did not include rare cancer diseases or rare infectious diseases that are outside the scope of the National Clinical Programme for rare diseases.

Each participating GP was surveyed as to which resources (online/print) they would use to inform themselves when faced with a patient with a rare disease, as well as their knowledge and use of Orphanet.

Each GP was provided with questionnaires to complete for each patient they identified with one of the 22 surveyed rare diseases. This anonymised questionnaire included questions on (i) RD diagnosis; (ii) demographics (age group, gender, age of diagnosis, health insurance status); (iii) time demand (frequency of consultations per year, reasons for attending, type 
Table 1 Estimated European prevalence rates of the 22 selected Rare Diseases

\begin{tabular}{ll}
\hline Rare disease name & Estimated prevalence (per 100,000) \\
\hline Congenital isolated hyperinsulinism & $2.0 \mathrm{BP}$ \\
Scleroderma (systemic sclerosis, localised sclerosis) & $42.0 \mathrm{P}$ \\
Familial QT syndrome (Inc. Romano Ward) & $40.0 \mathrm{BP}$ \\
Primary systemic amyloidosis & $30.0 \mathrm{P}$ \\
Retinitis pigmentosa & $26.7 \mathrm{P}$ \\
Fragile X & $32.5 \mathrm{P}$ \\
Neurofibromatosis 1 & $21.3 \mathrm{P}$ \\
Marfan syndrome & $15.0 \mathrm{P}$ \\
Sickle cell anaemia & $22.0 \mathrm{P}$ \\
Sarcoidosis & $12.5 \mathrm{P}$ \\
Haemophilia & $7.7 \mathrm{P}$ \\
Huntington's disease and rare neurodegenerative disease & $2.7 \mathrm{P}$ \\
Phenylketonuria & $10.0 \mathrm{BP}$ \\
22q11.2 Deletion syndrome (DiGeorge syndrome) & $37.5 \mathrm{BP}$ \\
Duchenne or Becker muscular dystrophy & $4.78 \mathrm{P} / 1.53 \mathrm{P}$ \\
Prader-Willi syndrome & $3.1 \mathrm{BP}$ \\
Epidermolysis bullosa & $2.2 \mathrm{BP} *$ \\
Mucopolysaccharidosis (type 2) & $10.0 \mathrm{P}$ \\
Rare ataxias (including Friedreich's) & $2.0 \mathrm{P} *$ \\
Tuberous sclerosis & $12.0 \mathrm{P}$ \\
Osteogenesis imperfecta & $10.0 \mathrm{P}$ \\
Motor neuron disease & $3.85 \mathrm{P}$ \\
\hline
\end{tabular}

Estimates provided from the Orphanet Report Series

$P=$ prevalence; $B P=$ birth prevalence

*Estimates shown are for epidermolysis bullosa simplex and Friedreich's Ataxia of consultations (face-to-face contact, phone calls and home visits)); (iv) the GP role(s) in care of the patient; (v) the GPs' role in relation to the management of the patient; and (vi) the patient's attendance at specialist appointments. Participants were asked to anonymize patient data with a random fourdigit identifier prior to the questionnaire return to ensure patient confidentiality.

About 23 primary care centres of varying sizes (ranging from single practitioner to large practices) were invited via letter, email and telephone contact to participate. The practices were selected on a convenience sampling.

\section{Data collection and analysis}

A designated staff member from each primary care/GP centre reviewed records and provided anonymised data to the research team.

Data were entered into a Microsoft Excel spreadsheet, and percentages, medians and interquartile ranges were calculated. Calculations were stratified by presence or absence of statebased care. Tests of significance were calculated using the two-sided Wilcoxon-Mann-Whitney test [16].

\section{Results}

\section{Participating primary care centres}

Ten primary care centres of the invited 23 centres participated in this survey. 31 GPs from these centres completed the surveys. 11 out of the 13 centres that did not take part indicated interest in participation but were unable due to clinic time pressures, and 2 did not indicate why they were unwilling to participate. The practices were located across four different counties, with staff ranging from one to approximately 7.5 whole-time GPs.

\section{Completion of questionnaire}

Additional information was provided anecdotally by the GPs to the survey team during their visit. Data about rare disease diagnoses were not easily retrievable from their practice electronic records, and disease names and ICD-10 codes were of little assistance as search terms to identify RD patients. The practice IT systems used were reliant on ICPC-2 coding, International Coding on Primary Care, which is a patient problem-focused coding system and was not searchable by 
either the underlying rare disease diagnosis or ICD-10 code. ICD-9 was used in few of general practices. Retrieval of patients with the 22 diagnoses was frequently dependent on physician memory recall.

\section{Patient demographics}

Data on $174 \mathrm{RD}$ patients were returned from 10 primary care centres. About 171 patients had one of the 22 pre-identified common multi-systemic RDs (Table 2). Data were excluded for three patients whose diagnoses were not on the preidentified RD list. The most common recorded RD was sarcoidosis $(n=63,36.8 \%)$ followed by familial Long QT syndrome $(n=11,6.4 \%)$, phenylketonuria $(n=10,5.9 \%)$, scleroderma $(n=10,5.9 \%)$, motor neuron diseases $(n=9,5.3 \%)$ and rare neurodegenerative diseases $(n=8,4.7 \%)$. About $54 \%(n=92)$ of patients were male, and $46 \%(n=79)$ were female. Most were adults $(n=141,82 \%$ of the total) at the time of the survey. The age of diagnosis of the RD was adulthood in 69\% ( $n=118)$ of the patients. Around 57\% ( $n=97)$ of the RD patients had state-reimbursed medical care, and the remaining $43 \%$ were private fee-paying patients.

\section{Rare disease resources}

The most commonly used resources by the GPs $(n=31)$, in order of decreasing popularity, were GP Notebook $(17 \%)$, Google (15\%) and specialist letters (12\%) (see Table 3). Although 11 of the GPs surveyed (35.5\%) were aware of Orphanet, only 4 GPs (12.9\%) had used this in their practice.

Table 2 Demographic features of the 171 Irish rare disease patients (2013-2017) surveyed from ten primary care practices

\begin{tabular}{lcc}
\hline Variable & \multicolumn{2}{c}{ Rare disease patients $N=171$} \\
\cline { 2 - 3 } & $N$ & $(\%)$ \\
\hline Gender & & \\
Male & 92 \\
Female & 79 & \\
Age (years) at time of survey & & \\
$\geq 18$ & 141 \\
$<18$ & 30 \\
Age (years) when diagnosed with $\mathrm{RD}(N=171)$ \\
$\geq 18$ & 118 \\
$<18$ & 53 & \\
Healthcare funding eligibility & & \\
State-reimbursed care & 97 \\
Not state-reimbursed & 74 & $(18)$ \\
\hline
\end{tabular}

$n=$ number of patients.

Ages ranged from 3 to 85 years old
Table 3 Preferred resources used by 31 surveyed Irish general practitioners to source rare disease information (2013-2017)

\begin{tabular}{lll}
\hline Resource(s) used & \multicolumn{2}{l}{ General practitioners $N=31$} \\
\cline { 2 - 3 } & $N$ & $\%$ \\
\hline GP Notebook & 15 & $(17)$ \\
Google & 13 & $(15)$ \\
Specialist letter & 11 & $(12)$ \\
Patient.co.uk & 10 & $(11)$ \\
Medscape & 9 & $(10)$ \\
Royal College Hospital in Melbourne App & 8 & $(9)$ \\
Registered Charities & 8 & $(9)$ \\
Information from patient & 8 & $(9)$ \\
British Medical Journal Best Practice & 4 & $(4)$ \\
Journals & 3 & $(3)$ \\
\hline
\end{tabular}

\section{Patient attendance rates}

The median attendance for all RD patients $(n=171)$ to GPs over the 4-year survey period was 16 (IQR $=8-33$ ) or 4.0 visits per annum. Around $88.7 \%$ of these consultations were face-to-face, $11 \%$ were phone calls and $0.2 \%$ were home visits. When stratified by payment method, those with state-reimbursed care visited their GP significantly more frequently than those paying privately (median number of visits 23 (IQR $=13-37)$ compared to 10 (IQR $=4-19)$, respectively $(p<0.005)$ ). There was no significant difference in type of consultation between the two payment groups.

\section{General practitioners' role in patient care}

Around 19\% $(n=32)$ of patients had their RD diagnosed by their GP, and for a further $19 \%(n=32)$, the GP played a major role in the diagnosis. Over $56 \%$ of patients $(n=96)$ obtained repeat prescriptions for their RD from their GP, 37\% $(n=63)$ had complications of their RD managed by their GP and $16 \%(n=28)$ were seen for routine RD management. Other reasons for attendance $(n=38,22 \%)$ included taking bloods prior to specialist appointments, provision of psychological support and management of RD in pregnancy. Of the 147 patients actively managed at the time of survey completion, $63 \%(n=93)$ of the patients had RD management only provided by specialists, $6 \%(n=9)$ had the RD managed solely by their GP and $16 \%(n=23)$ had their RD jointly managed by both a GP and a specialist. For the remaining $15 \%(n=22)$, it was unclear who was managing the patient's RD or whether the patient had been discharged from all services. 


\section{Discussion}

In this pilot survey, we have investigated the Irish general practitioners' role in the care of a selected number of rare diseases and rare diseases information/educational tools used by GPs. We observed that RD patients who were eligible for statereimbursed GP care $(n=97)$ had over twice the number of consultations (5.8 per year) compared to those who paid privately $(n=74)$ ( 2.5 per year). As the state-reimbursed category included means-tested and non-means-tested state reimbursement, it is difficult to draw any income-related conclusions from this data. However, as the cost burden has been alleviated for these patients, it could mean that they are more likely to attend their GP for guidance, management and support.

We found that $69 \%$ of RD patients surveyed were diagnosed in adulthood. This is a striking disparity considering $75 \%$ of RDs start in the paediatric period [4]. This may be accounted for by a bias of the selection of the diseases for the survey; 8 of the 22 diseases are exclusively of adult onset. Those conditions with adult onset have higher prevalence reported than the paediatric onset diseases surveyed (see Table 1). This pilot survey could not estimate the overall burden of RDs in general practice, as the survey included only 22 of the over 6000 RDs.

Our survey highlights the diverse roles of GPs in RD patient care, ranging from diagnostics to management of RD complications. For $38 \%$ of patients their GP was involved in their diagnosis either by making the diagnosis directly or playing a major role in the diagnostic work up of the patient. This compares to a recent Belgian study which showed that, for $121 \mathrm{RD}$ patients, $36 \%$ of the time the GP established the diagnostic referral [17].

Our data suggest that most of the patients had their RD managed primarily by specialists, while some GPs were unaware of whom was the primary physician managing the patient. These data correspond with the Belgian experience which reported that GPs chose to refer RD patients to specialists rather than directly consulting the specialists to help reach a diagnosis [18]. Patients who are managed entirely by specialists alone may miss out on support and allied health services that are available at a local level.

The GPs in this survey did not generally use Orphanet. Only $35.5 \%$ of GPs surveyed were aware of Orphanet and an even lower percentage of users surveyed (12.9\%) use this resource, comparable to the Belgian study, which showed that Orphanet was used by only 10 of the 64 (16\%) GP practices with RD patients [18]. Utilisation of Orphanet would be helpful to GPs as it contains centralised, curated and current information about Irish rare disease resources. Other popular resources among GPs may provide a general overview of RDs, but they offer little information beyond this, such as details on pathophysiology, locations of centres of expertise, support groups, current research and clinical trials.
Both our survey and the Belgian study point to the lack of a specific RD coding system used in general practice. GPs have difficulty identifying the RD patients in their practices through use of the electronic system, often relying on memory. The use of a specific RD coding system, such as ORPHAcodes, as part of the patient's electronic health record would allow for the burden of RDs in general practice to be measured [3]. Such an approach is being adopted elsewhere: in England, the move to a single terminology, SNOMED CT, was implemented across primary care and began to be deployed to GP in a phased approach from April 2018 [19, 20]. Currently in Ireland, the implementation of the SNOMED CT has been agreed for retrofit to GP software, which will facilitate more widespread and accurate recording of rare diseases. However, the EU Commission 2009 recommendations regarding coding, recently reiterated by the EC Steering Group on Health Promotion, Disease Prevention and Management of Non-Communicable Diseases (SGPP) Committee, has recommended adding ORPHAcodes to national health information systems [21].

Access to a shared electronic health record could improve communication and enhance patient care by clarifying which health care providers are actively managing the patient and allowing co-ordination of care between primary and specialist care. Ireland is currently lacking a shared electronic health record system linking hospitals and primary care centres. This initiative is planned for the proposed Slaintecare health programme to parallel the Northern Ireland Electronic Care Record (NIECR) [22]. A shared record would allow RD codes to be assigned to the patient at diagnosis and to remain associated with the patient record so repeat coding for chronic conditions would not be required.

\section{Survey limitations}

Recruitment of centres for this survey proved to be a challenging task, reflected in the poor response rate. The resulting major limitation of this survey is the small sample of patients due to the small number of participating GPs. The reliance of physician memory recall in many instances to identify subjects with one of 22 rare diseases may also have led to under-ascertainment of patients in this survey. Without Irish reference data on the prevalence of individual rare diseases, it is not possible to test whether the expected number of cases of each diagnosis was obtained in this survey or assess the degree of under-ascertainment. In the absence of centralised electronic health records, it is not possible to track RD patients that may have left the participating GP practices or who may have died over the survey period.

As a pilot survey, it does however shed some light into the RD patient journey involving primary health care. A larger survey would be necessary to provide an adequate sample size to generate sufficient data for rigorous statistical analysis. However, the feedback from the non-participating practices indicated that busy competing clinical pressures limited the 
ability to retrieve and collate data, greatly compounded by inadequate primary care IT and coding systems for RDs.

\section{Conclusions}

In conclusion, this survey indicates that management for RD patients in Ireland is more likely to be managed by specialist consultants than in primary care.

Further evaluation of the two-way communication between primary care and specialist health care services is warranted given that there is on occasion uncertainty around which physician leads the management in the care of some patients with rare diseases. Introduction of RD coding nomenclature and shared electronic health records would permit RD patients to be identified within primary care systems to enable future RD healthcare planning and facilitate epidemiological research. It is hoped in the future that enhanced education in primary care and use of the existent national RD educational resources (www.rarediseases.ie) and Orphanet (www.orpha.net) will assist with improving the coordination and care of RD patients in the community settings.

Acknowledgments Dr. David Hanlon (MICGP) is thanked for his helpful discussion at the conceptualisation stage of this survey.

\section{Compliance with ethical standards}

Conflict of interest The authors declare that they have no conflicts of interest.

Ethical approval As retrospective anonymised aggregate practice (community) attendance data was used in this survey with no direct patient contact, institutional research ethics committee approval was not obtained/deemed necessary for this survey.

Open Access This article is licensed under a Creative Commons Attribution 4.0 International License, which permits use, sharing, adaptation, distribution and reproduction in any medium or format, as long as you give appropriate credit to the original author(s) and the source, provide a link to the Creative Commons licence, and indicate if changes were made. The images or other third party material in this article are included in the article's Creative Commons licence, unless indicated otherwise in a credit line to the material. If material is not included in the article's Creative Commons licence and your intended use is not permitted by statutory regulation or exceeds the permitted use, you will need to obtain permission directly from the copyright holder. To view a copy of this licence, visit http://creativecommons.org/licenses/by/4.0/.

\section{References}

1. Angelis A (2015) Socio-economic burden of rare diseases: a systematic review of cost of illness evidence. Health Policy (Amsterdam, Netherlands) 119(7):964-979

2. Annemans L, Aymé S, Le Cam Y et al (2017) Recommendations from the European working Group for value assessment and funding processes in rare diseases (ORPH-VAL). Orphanet $\mathrm{J}$ rare dis 12(1):50

3. Aymé S, Bellet B, Rath A (2015) Rare diseases in ICD11: making rare diseases visible in health information systems through appropriate coding. Orphanet J Rare Dis 10:35

4. Nguengang Wakap S, Lambert DM, Olry A, Rodwell C, Gueydan C, Lanneau V, Murphy D, Cam Y, Rath A (2019) Estimating cumulative point prevalence of rare diseases: analysis of the Orphanet database. Eu J Hum Genet, in press:1-9. https://doi.org/10.1038/ s41431-019-0508-0

5. Eurordis 2 (ed.) (2009) The voice of 12,000 patients: Eurordis. https://www.eurordis.org/IMG/pdf/voice_12000_patients/ EURORDISCARE_FULLBOOKr.pdf. Accessed November 2019

6. Grier J, Hirano M, Karaa A et al (2018) Diagnostic odyssey of patients with mitochondrial disease: results of a survey. Neurol genet 4(2):e230

7. Global commission to end the diagnostic odyssey for children with a rare disease: February 28, 2019. https:// globalrarediseasecommission.com accessed November 2019

8. Hayward J, Bishop M, Rafi I, Davison V (2017) Genomics in routine clinical care: what does this mean for primary care? $\mathrm{Br} \mathrm{J}$ Gen Pract 67(655):58-59

9. EMPATHIE https://ehff.eu/wp-content/uploads/2015/10/ empathie_frep_en.pdf. Accessed November 2019

10. Starfield $\bar{B}(2012)$ Primary care: an increasingly important contributor to effectiveness, equity, and efficiency of health services. SESPAS report 2012. Gac Sanit 26(Suppl 1):20-26

11. Elliott E, Zurynski Y (2015) Rare diseases are a 'common' problem for clinicians. Aust Fam Physician 44(9):630-633

12. Anderson M, Elliott EJ, Zurynski YA (2013) Australian families living with rare disease: experiences of diagnosis, health services use and needs for psychosocial support. Orphanet J Rare Dis 8:22

13. Iacobucci G (2019) NHS long term plan: care to be shifted away from hospitals in "21st century" service model. BMJ 364:185

14. Slaintecare. https://www.gov.ie/en/campaigns/slaintecareimplementation-strategy. Accessed November 2019

15. Rath A, Olry A, Dhombres F, Brandt MM, Urbero B, Ayme S (2012) Representation of rare diseases in health information systems: the Orphanet approach to serve a wide range of end users. Hum Mutat 33:803-808

16. Marx A, Backes C, Meese E, Lenhof HP, Keller A (2016) EDISON-WMW: exact dynamic programing solution of the Wilcoxon-Mann-Whitney test. Genomics Proteomics Bioinformatics 14(1):55-61

17. Boffin N, Swinnen E, Wens J et al (2018) General practice care for patients with rare diseases in Belgium. A Cross-Sectional Survey. Int J Environ Res Public Health 15(6)

18. Boffin N, Moreels S, Van Casteren V. (2013) The Belgian network of sentinel general practices between 2007 and 2012: a short report. Brussels: Wetenschappalijk Institut Volksezonheid Institut Schentifique de saint publique ISP WIV

19. Cornet R, de Keizer N (2008) Forty years of SNOMED: a literature review. BMC Med Inform Decis Mak 8(Suppl 1):S2-S2. https:// doi.org/10.1186/1472-6947-8-S1-S2

20. Randorff Højen A, Gøeg KR (2012) SNOMED CT Implementation. Methods Inf Med 51(6):529-538. https://doi. org/10.3414/ME11-02-0023

21. EU Council 2009. Council Recommendation of 8 June 2009 (2009/C 151/02) on an action in the field of rare diseases: European Commission (ISSN 1725-2423)

22. Northern Ireland Electronic Care Record (NIECR). http://www. ehealthandcare.hscni.net/niecr/niecr.aspx Accessed November 2019

Publisher's note Springer Nature remains neutral with regard to jurisdictional claims in published maps and institutional affiliations. 